\title{
A TWO STEP METHOD FOR RECORDING HOLOGRAPHIC OPTICAL ELEMENTS WITH PARTIALLY COHERENT LIGHT
}

\author{
I. PASCUAL, A. BELÉNDEZ, A. FIMIA
}

KEY WORDS :

Holograms copying

Partially coherent light
MótS Clés :

Copie d'hologrammes

Lumière partiellement cohérente
SUMMARY : We propose a two step method which enables us to obtain transmission Holographic Optical Elements of high diffraction efficiency as well as a good signal-to-noise relation. By means of a device using partially coherent light, a master Holographic Optical Element can be copied in a second photosensitive material. The results which are obtained in dichromated gelatin and photoresist are comparable to those which are obtained directly.

\begin{abstract}
Méthode à deux étapes pour la réalisation d'éléments optiques holographiques en lumière partiellement cohérente
\end{abstract}

RÉSUMÉ : Nous présentons une méthode en deux étapes qui permet de fabriquer des éléments optiques holographiques en transmission avec un rendement de diffraction élevé et un bon rapport signal sur bruit. Nous partons d'un élément optique holographique original, et moyennant un système en lumière partiellement cohérente, cet original est copié sur un second matériel photosensible. Les résultats de rendement de diffraction obtenus, avec cette méthode, sur gélatine bichromatéc et sur photorésine sont comparables avec les résultats obtenus directement.

\section{1. - INTRODUCTION}

There are, in Holography, many recording materials, the spectral response of which has high chromatic sensitivity in short wavelength zones. Both dichromated gelatins and photoresist present a variation in sensitivity which can go from 1 to 100 when we pass from $514 \mathrm{~nm}$ to $405 \mathrm{~nm}$ [1]. Furthermore, in particular zones of the spectrum no laser sources can be found, which makes it impossible to use the chromatic sensitivity of these recording materials.

In order to overcome this disadvantage, in this paper we present a possible alternative for obtaining Holographic Optical Elements by transmision in the holographic recording media that we have mentioned above.

The method consists of copying a previously achieved Holographic Optical Element (HOE) in Fresnel's region [2] so that, once it has been placed in Bragg's angle, and once its object beam and the reference beam in reconstruction have been optimized so that they can have a 1 to 1 relation in the second stage, we will be able to copy, on a dichromated gelatin or photoresist layer, a second HOE, the diffraction efficiency of which is comparable to that obtained under normal circumstances.

In this second step we can work with partially coherent light, which enables us to work with more economical sources and devices, and provides stability conditions that are not as strict as those used in conventional holographic devices.

Previous studies have proven the usefulness and possibilities of this method in obtaining diffusse objects that have high diffraction efficiency and a good signal-to-noise relation [3]. 
In this paper we will also discuss the calculation of the coherence conditions of the experimental device, the experimentation process itself and the results we obtained.

\section{2. - CAlCUlation}

\section{OF THE COHERENCE FACTOR}

In figure 1 we have schematized the geometry that is proposed for obtaining Holographic Optical Elements by copying in Fresnel's region. The master, a diffraction grating or a holographic lens, is placed in contact with the second recording medium on which we are going to copy. We make use of both the transmitted beam and the diffracted +1 , which both interfere on the second medium, making sure by means of the volume effects, that there are no secondary diffraction orders which could give rise to parasite images. This geometry and the contact between the original and the copy allow us to use an extensive and partially coherent light source.

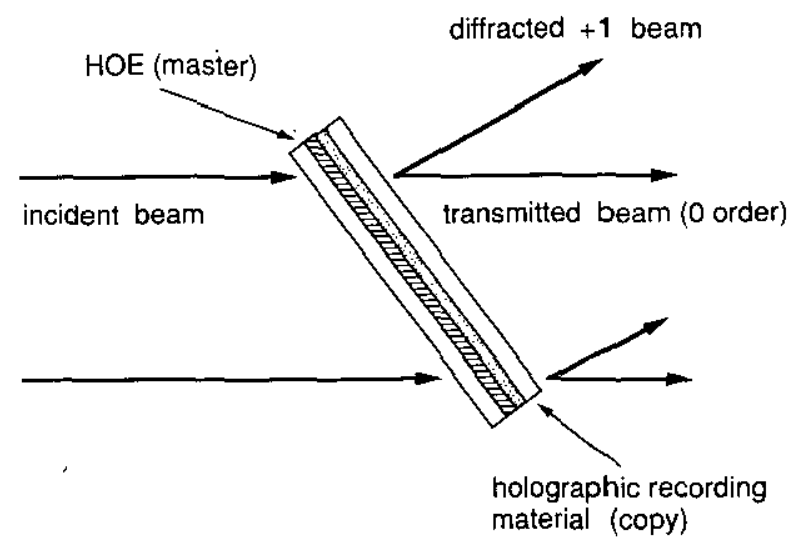

FJG. 1. - Geometry proposed for obtaining holographic optical elements by copying in Fresnel's region.

Let us consider the situation which is schematized in figure 2. The quasimonochromatic light which comes from an extensive source $S$ reaches points $P_{1}$ and $P_{2}$ by means of an ordinary optical system [4]. The degree of coherence between the optical vibrations in $P_{1}$ and $P_{2}$ will determine the modulation of the interference fringes in $P$, where both will overlap.

Irradiance in $P$ may be written as :

$$
E=E_{0}+E_{1} \cdot \operatorname{Re}\{\xi\} .
$$

The factor of total coherence, $\mathfrak{F}$ - except in a constant phase factor - is the product of the spatial coherence factor $E$ and the time coherence factor $T$, so that we can write :

$$
y=\exp \quad\left\{\mathrm{i} 2 \pi \delta / \lambda_{0}\right\} E . T
$$

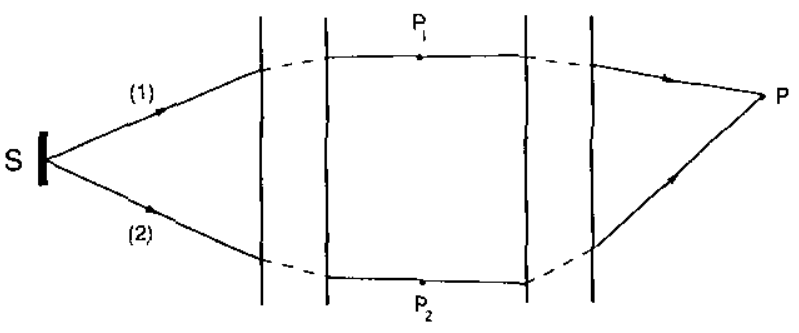

FIG. 2. - The spatial coherence between two points.

where $\lambda_{0}$ is the wavelength used and $\delta$ is the total path difference between the source and point $P$ where the beams interfere. When the source is circular and is illuminated in a uniform way, VanCittert-Zernike's theorem gives us the relation for the time coherence factor [5]:

$$
E=\frac{2 J_{1}(v)}{v}
$$

where $v$ is :

$$
v=\frac{2 \pi r \phi}{\lambda_{0}}
$$

In these equations $J_{1}(v)$ is the Bessel function of the first kind of index one, $\phi$ is the angular size of the source and $" r$ " is the distance between $P_{1}$ and $P_{2}$ (fig. 3).

If we now analyze the geometrical disposition of both layers of recording material as well as that of the layer of air which remains between them (fig. 4) we find that the distance between $P_{1}$ and $P_{2}$ can be expressed as approximately:

$$
r \approx h \cdot \operatorname{sen} \theta^{\prime}
$$

where $h$ is :

$$
h=h_{1}+a+h_{2} \text {. }
$$

In these equations $h_{1}$ and $h_{2}$ are the thicknesses of the master and copy, respectively, $\langle a »$ is the thickness of the air layer between the primary hologram and the copy and $\theta^{\prime}$ is Bragg's angle in the recording medium. From this figure we find that :

$$
\begin{aligned}
\delta=h_{1} n_{1}\left(1-\cos \theta^{\prime}\right)+h_{2} n_{2}\left(1-\cos \theta^{\prime}\right)+ \\
+a(1-\cos \theta)
\end{aligned}
$$

which is nothing but the difference of path between the ray of light which goes through $P_{1}$ and $P_{2}$ and finally reaches $P$. In this equation, $n_{1}$ and $n_{2}$ are the refractive index of the master and copy, respectively.

Moreover as the spectral distribution of source is gaussian and finite, the time coherence factor is given by [4] :

$$
T=\exp \left\{-\pi\left(\Delta \lambda^{\prime} \delta / \lambda^{\prime 2}\right)^{2}\right\}
$$




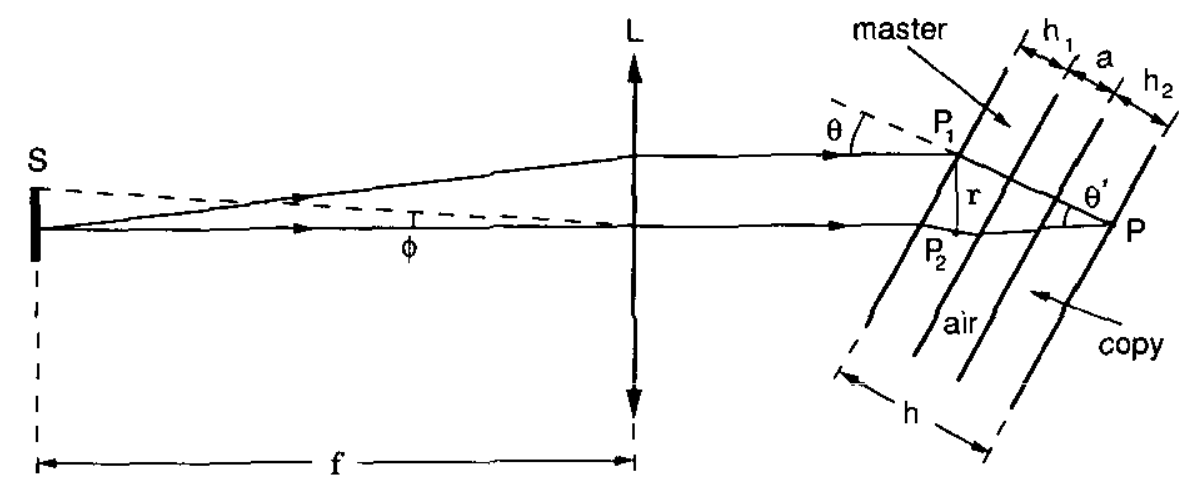

FIG. 3. - Experimental arrangement used to evaluate spatial and temporal coherence.

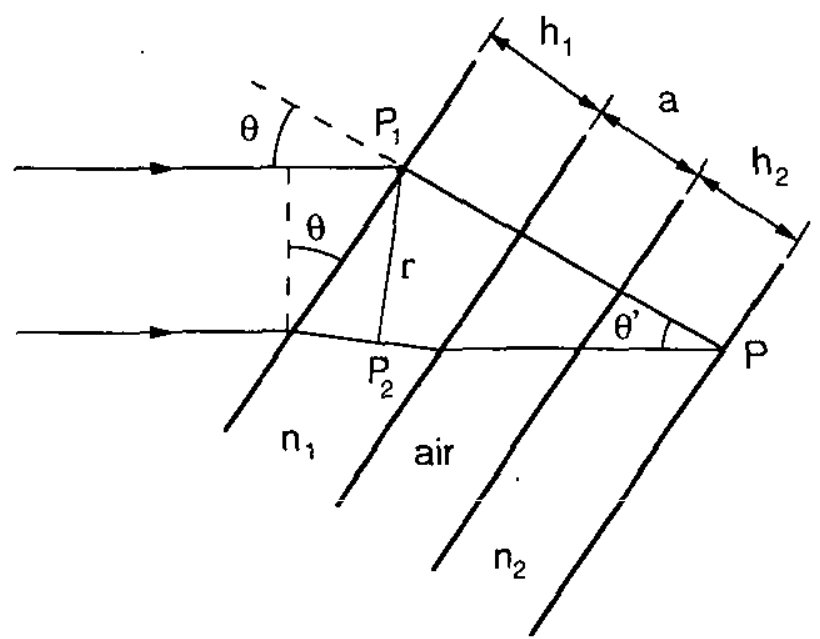

FIG. 4. - Geometrical disposition of both layers of recording material and the layer of air which remains between them.

where $\Delta \lambda^{\prime}$ is the bandwidth of the points where the intensity of the source has descended to a value of $\exp (-\pi / 4)$ times its maximum value in the emission line, and $\delta$ is the total difference of path that has been previously calculated.

In the course of our experiments we have used a Philips CS150W high pressure mercury lamp. In order to study time coherence, we isolated the emission lines with interference filters that have a bandwidth of $12 \mathrm{~nm}$. Using a Michelson's interferometer, we measured the variation in visibility of the interference figure, which gave us the variation of the optical path for the different mercury emissions. The values of $\delta$ that have been measured using this method are shown in table $I$ and correspond to fringe visibility values superior or equal to $80 \%$ [6].

From the results of the measurement of $T(\delta)$, we deduce that $405 \mathrm{~nm}$ is the most adequate radiation
TABLE 1

$V$ alues of $\delta$ measured with a Michelson's interferometer.

\begin{tabular}{|l|c|c|}
\hline Lamp & $\begin{array}{c}\text { Interference tilter } \\
(\Delta \lambda=12 \mathrm{~nm})\end{array}$ & $\delta(\mu \mathrm{m})$ \\
\hline High-pressure Hg & $578 \mathrm{~nm}$ & 7 \\
Philips CS150W & $546 \mathrm{~nm}$ & 9 \\
$436 \mathrm{~nm}$ & 4 \\
$405 \mathrm{~nm}$ & 7 \\
$365 \mathrm{~nm}$ & 2 \\
\hline
\end{tabular}

linc for our purposes, since, although $578 \mathrm{~nm}$ and $546 \mathrm{~nm}$ present bigger differences of path, the recording materials which we wish to work with are not sensitive to these radiations.

As our master has been made in a silver halide sensitized gelatin [7], from Agfa-Gevaert 8E56 HD with a nominal thickness of $5 \mu \mathrm{m}$, and since the thickness of the Kodak $649 \mathrm{~F}$ plate that was used for the copy was $15 \mu \mathrm{m}$, and taking into account a separation between both emulsions of $3 \mu \mathrm{m}$ (measured by interference method [8]), the numerical values for the calculations of the rates of coherence are presented in table II. These results are consistent with the experimental results that have been obtained as we are going to discuss in the following section.

\section{TABLE II}

Numerical values of the rates of coherence for an master with a nominal thickness of $5 \mu \mathrm{m}$, a copy of $15 \mu \mathrm{m}$, and taking into account a separation between both emulsions of $3 \mu \mathrm{m}$.

\begin{tabular}{|c|c|}
\hline$\lambda(\mathrm{nm})$ & $\begin{array}{c}\delta_{\text {experimental }}(\mu \mathrm{m}) \\
(\Delta \lambda=8.2 \mathrm{~mm})\end{array}$ \\
\hline 405 & 30 \\
436 & 20 \\
546 & 40 \\
578 & 30 \\
\hline
\end{tabular}




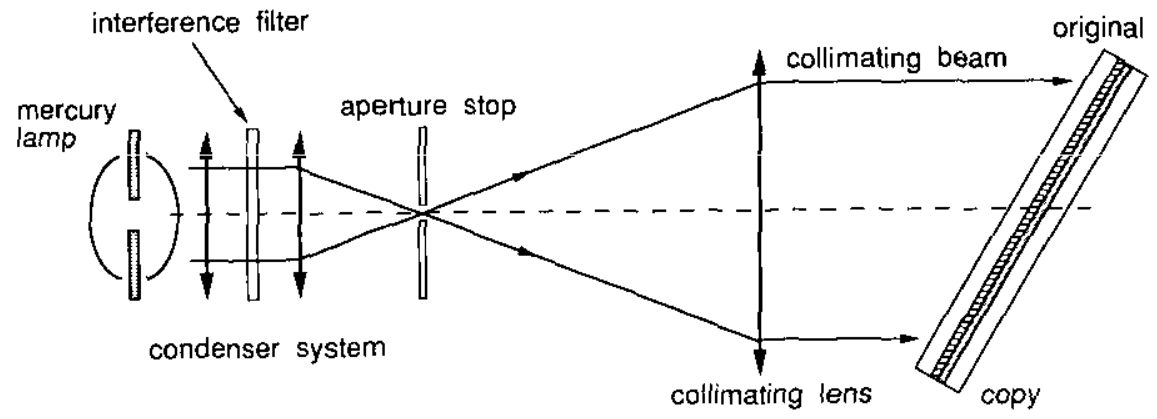

FIG. 5. - Basic scheme of the experimentul device used to copy holographic optical elements with partially coherent light.

\section{3. - EXPERIMENTS THAT HAVE BEEN PERFORMED}

Figure 5 represents our experimental arrangement. The radius of the source was $30 \times 10^{-3} \mathrm{rad}$, and the quantity of light which we had at our disposal was $40 \mu \mathrm{W} / \mathrm{cm}^{2}$. These conditions led us to one-hour-long exposition periods; nevertheless, stability conditions were very good because since the master and the copy were in close contact, any mechanical perturbation affected them equally.

The masters were made in silver halide sensitized gelatin, because this processing presents a high signal-to-noise relation (SNR), with stability and good diffraction efficiency in almost the whole visible spectrum. We made diffraction gratings of 1000 lines $/ \mathrm{mm}$ on the Agfa 8E56 HD plate, the exposition being adjusted in order to obtain a diffraction efficiency of $40 \%$ in the $405 \mathrm{~nm}$ radiation used. These conditions enabled us to have a one to one relation between the 0 order and the diffracted +1 on the medium which was to be exposed in the second stage. Thanks to this, we were able to obtain maximum diffraction efficiency in the copy.

\section{4. - RESULTS}

In figure 6 we have a representation of diffraction efficiency versus the exposure for gratings copied in photoresists. As can be seen, we reached efficiencies of $40 \%$, superior to the theoretical ones if we take into account that the recording material acts as a thin hologram. However, the profiles may not be sinusoidal in this case [9], a fact which explains these results. Table $1 / I$ shows the process of obtaining photoresist layers and the developing process that has been used.

In figure 7 we likewise offer a representation of the results for dichromated gelatin. We have obtained results in diffraction efficiency of $75 \%$ in the gratings. The processing is the one used by Meyerhofer [1], except that the concentration of

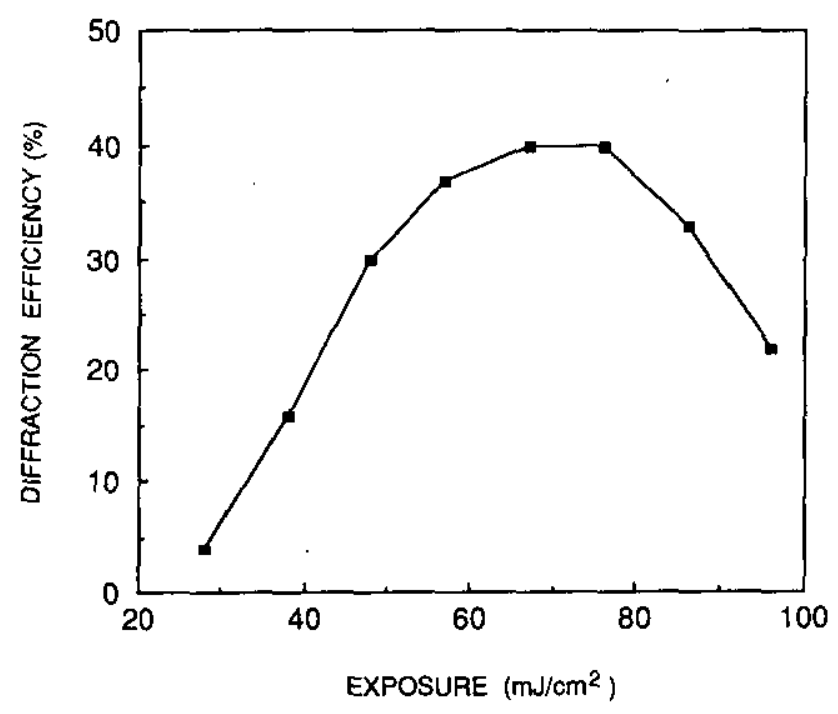

FIG. 6. - Diffraction efficiency versus exposure for gratings copied in photoresists.

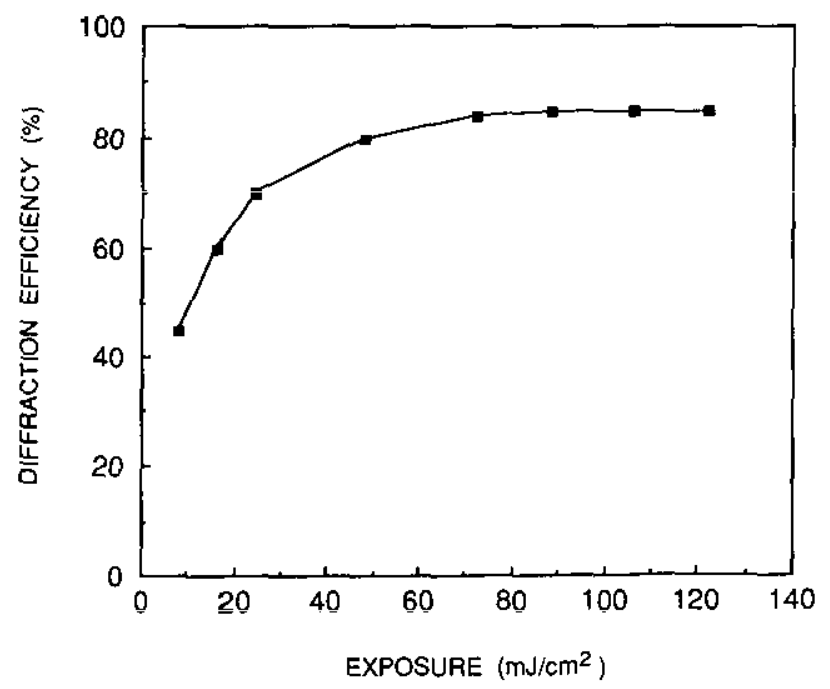

FIG. 7. - Diffraction efficiency versus exposure for gratings copied in dichromated gelatin. 
TABLE III

Process for abtaining photoresist layers and the developing process used.

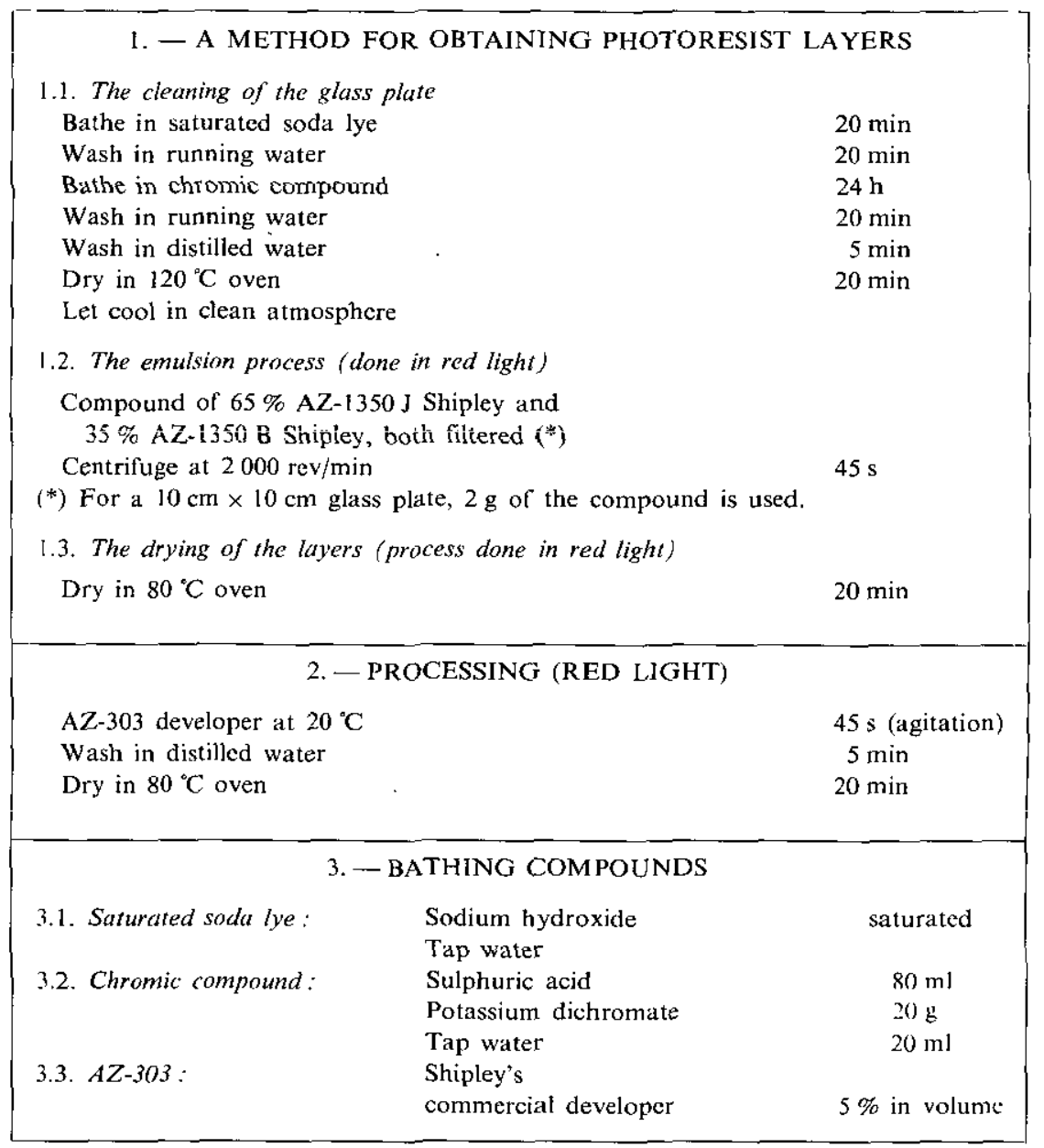

dichromate used was $0.5 \%$. This is due to the absorption coefficient of the gelatin layers in the $405 \mathrm{~nm}$ zone. In figure 8 we present the measurements of the absorption coefficient $(\alpha)$ as a function of the concentration of ammonium dichromate for

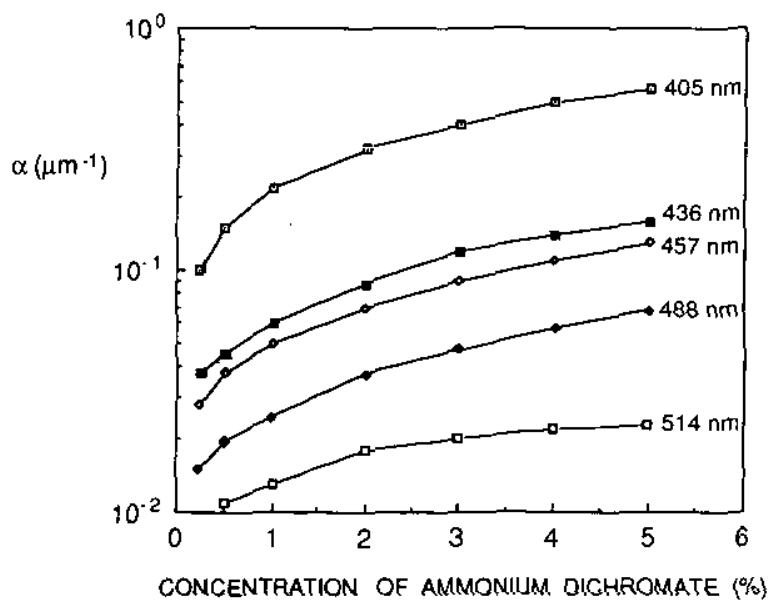

FiG. 8. - Measturements of the absorption coefficient ( $\alpha$ ) of the gelatin layers versus the concentration of ammonium dichromate for different wavelengths. the different wavelengths. As can be seen, it is necessary to reduce the concentration to $0.5 \%$, so that the radiation can act in a uniform way on the whole volume of the recording material.

Once the optimum energies of work were obtained from this data, they were copied on the holographic lens that we have described above, thereby obtaining high diffraction efficiencies and uniformities on the whole opening of the lens, both in photoresist and in dichromated gelatin.

Although the exposure periods are long, we have to bear in mind, that we are using the high sensitivity which these materials present in short wavelength zones.

\section{5. - CONCLUSIONS}

The two step method that we have just presented here allows us to obtain diffraction gratings and holographic lenses by transmission in dichromated gelatins and photoresists, with diffraction efficiencies that are comparable to the ones we obtained with direct methods. Likewise, it enables us to work with 
partially coherent light sources, by which it is possible to obtain holographic optical elements in areas of the spectrum where no adequate lasers exit, thereby taking advantage at the same time of the high energetic sensitivity which the recording materials present in the short wavelength zones of the spectrum.

It also permits us to obtain great series of holographic optical elements with similar characteristics in a simple and economical way.

\section{REFERENCES}

[1] Meyerhofer (D.). - «Dichromated Gelatin" in Holographic Recording Materials, H. M. Smith, Editor. Springer-Verlag, Berlin 1977, pp. 75-99.

[2] VANiN (V. A.). -- Hologram Copying (Review). Sov. $J$. Quantum Electron., 1979, Vol. 8, 809-818.
[3] Oliva (J.), Fimia (A.), Quintana (J. A.). - Diffuse-Object Holograms in Dichromated Gelatin. Appl. Opt., 1982, Vol. 21, 2891-2893.

[4] HoPkINS (H. H.). - «The Theory of Coherence and its Applications" in Advanced Optical Techniques, A.C.S. Van Heel, Editor. North Holland, Amsterdam 1967, pp. 189-237.

[5] Born (M.), Wolf (E.). - Book "Principles of Optics". Pergamon Press, Oxford 1987, pp. 491-554.

[6] Fimia (A.). - " Copia de Hologramas con Luz Parcialmente Coherente ». Ms. Degree Thesis. University of Vaiencia, Valencia 1980

[7] Fimia (A.), Pascual (I.), Vázquez (C.), Belendez (A.). Silver-Halide Sensitized Holograms and their Applications, 1989. Proceeding SPIE, Vol. 1136, 53-57.

[8] Altman (J: H.), - Method for measuring the absolute separation between glass plated during contact printing. Phot Science and Engineering, 1970, Vol. 14, 428-431.

[9] EnGer (R. C.), CASE (S. K.). « High-Frequency Holographic Gratings in Photoresist». J. Opt. Soc. Am. A, 1983, Vol. $73,1113-1118$.

(Manuscript received in February 2, 1991.) 